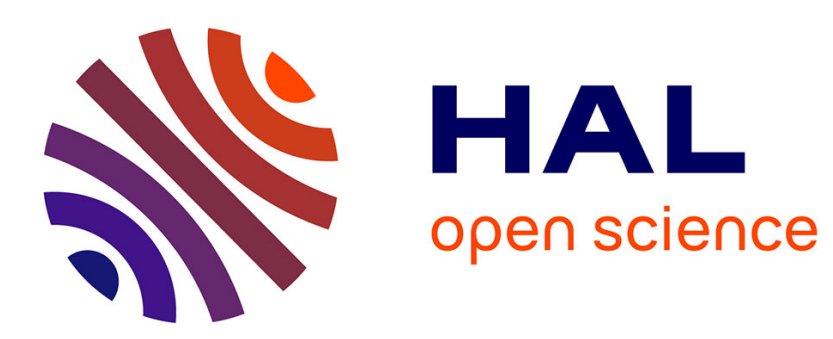

\title{
Mean covariogram of cylinders and applications to Boolean random sets
}

\author{
François Willot
}

\section{To cite this version:}

François Willot. Mean covariogram of cylinders and applications to Boolean random sets. Journal of Contemporary Mathematical Analysis, 2017, 52 (6), pp.305-315. 10.3103/S1068362317060061. hal-01678545v2

\section{HAL Id: hal-01678545 \\ https://hal.science/hal-01678545v2}

Submitted on 6 Nov 2018

HAL is a multi-disciplinary open access archive for the deposit and dissemination of scientific research documents, whether they are published or not. The documents may come from teaching and research institutions in France or abroad, or from public or private research centers.
L'archive ouverte pluridisciplinaire HAL, est destinée au dépôt et à la diffusion de documents scientifiques de niveau recherche, publiés ou non, émanant des établissements d'enseignement et de recherche français ou étrangers, des laboratoires publics ou privés. 


\title{
Mean covariogram of cylinders and applications to Boolean random sets
}

\author{
F. Willot ${ }^{1, *}$ \\ ${ }^{1}$ Mines ParisTech, PSL Research University, \\ 35 rue $S^{t}$-Honoré, 77300 Fontainebleau, France.
}

This work focuses on the variance properties of isotropic Boolean random sets containing randomly-oriented cylinders with circular cross-section. Emphasis is put on cylinders with large aspect ratios, of the oblate and prolate types. A link is established between the powerlaw decay of the covariance function and the variance of the estimates of the volume fraction of cylinders. The covariance and integral range of the Boolean mixtures are expressed in terms of the orientation-averaged covariogram of cylinders, for which exact analytical formulas and approximate expressions are provided.

MSC numbers: 60D05; 52A22; 53C65

Keywords: Stochastic geometry; Geometrical covariogram; Boolean model; Cylinder

\section{INTRODUCTION}

The covariogram, originally introduced by G. Matheron [13], gives the volume of the intersection of a (convex) body with a translation of itself. The function is closely related to the distribution of the length of the chords of a body, and also a key ingredient in the theory of Boolean stochastic models based on Poisson point processes (see [2, 14, 16, 17, 22] and references therein). A key-theorem relates the covariance function of stationary Boolean sets in the Euclidean space to the Poisson intensity of the point process and to the covariogram of the primary grain. In turn, the covariance function itself governs basic features of the model, such as the specific surface area and the integral range. The integral range is linked to the estimates on finite-size volumes of the random set volume fraction.

Another property of interest concerns the probability that a segment is entirely contained in the complementary set of a Boolean model. This probability takes a simple form for convex primary grains, which depends on the derivative of the covariogram at 0 [22]. Linear erosion allows one to compute this probability numerically which is especially useful for model identification $[11,18]$.

An important subclass of Boolean models, commonly used in material science for modelling heterogeneous materials, concern isotropic random sets. In dimension 3, this model requires one to average the covariogram over all directions uniformly on the sphere. In the rest of this article, the orientation-averaged covariogram, also denoted "isotropized covariogram" in the litterature, will be refered to as "mean covariogram". The mean covariogram is a special case of so-called "kinematic" integrals (see [21], Chap. 5) of the form (in

*Electronic address: francois.willot@ensmp.fr 
dimension $d)$ :

$$
\int_{G^{d}} V_{d}(M \cap g A) \mu(\mathrm{d} g)
$$

where a convex body $A$ is moved into $g A$ and the volume of the intersection of $g A$ with a convex set $M$ is averaged with respect to the measure $\mu$ over all transformations $g$ in the motion group $G^{d}$. The measure $\mu$ is invariant in $G^{d}$. The mean covariogram required for describing isotropic Boolean models is obtained by taking translations at fixed distance uniformly distributed on the sphere for $G^{d}$ and $\mu$ and $M=A$. Unfortunately, such kinematic covariogram is usually not known explicitely, especially in 3D. Some notable exceptions include the sphere, parallelepiped, cylinder [6, 8] and Poisson polyhedra [15]. The mean covariogram for cylinders is useful for identifying stochastic fibrous models from experimental 2D (e.g. SEM) or 3D (e.g. tomography) images of fibrous materials [18-20]. More generally, heterogeneous microstructures studied for various industrial applications can be approached by random models of cylinders. Examples include flakes in optics [4] or platelets in mesoporous materials [24].

This work focuses on the mean covariogram of 3D cylinders with circular cross-section and its applications to Boolean models. It is organized as follows: the covariogram of a cylinder is recalled in Sec. (II). The mean covariogram is derived in Sec. (III). Boolean models of cylinders are considered in Sec. (IV). The integral range is given in Sec. (IV A). Variance properties are discussed in Secs. (IV B) and (IV C). We conclude in Sec. (V).

\section{COVARIOGRAM OF A CYLINDER}

Consider a cylinder $\boldsymbol{C}$ of height $h$ and circular cross-section of radius $r$, and a Cartesian coordinate system with origin $O$ and axes $\mathbf{e}_{x}, \mathbf{e}_{y}$ and $\mathbf{e}_{z}$. We assume that $O$ is at the center of one of the bases of $\boldsymbol{C}$ and that $\mathbf{e}_{z}$ is parallel to the cylinder main axis. Consider now the translation $\boldsymbol{C}^{\prime}$ of the cylinder $\boldsymbol{C}$ by a vector $\boldsymbol{v}$. We parametrize $\boldsymbol{v}$ by its norm $t=|\boldsymbol{v}|$ and two angles $\phi \in[0 ; 2 \pi]$ and $\theta \in[-\pi / 2 ; \pi / 2]$ in spherical coordinates. The azimuthal angle $\phi$ is the angle between $\mathbf{e}_{x}$ and the projection of $\boldsymbol{v}$ onto the plane $\left(0 ; \mathbf{e}_{x}, \mathbf{e}_{y}\right)$. The variable $\theta$ denotes the angle between $\boldsymbol{v}$ and the plane $\left(0 ; \mathbf{e}_{x}, \mathbf{e}_{y}\right)$ so that $\theta=\pi / 2$ when $\boldsymbol{v}$ is parallel to $\mathbf{e}_{z}$ and $\theta=0$ when $\boldsymbol{v}$ is contained in the plane $\left(0 ; \mathbf{e}_{x}, \mathbf{e}_{y}\right)$. Note that, using this convention, $\theta$ is the complementary of the polar angle.

The (oriented) covariogram of a cylinder $K(\theta, t)$ is defined as the volume of the intersection of $\boldsymbol{C}$ with $\boldsymbol{C}^{\prime}$ :

$$
K(\theta, t)=L_{3}\left(\boldsymbol{C} \cap \boldsymbol{C}^{\prime}\right),
$$

where $L_{3}$ denotes the Lebesgue measures in $\mathbb{R}^{3}$. The covariogram $K$ depends on $r, h, t$ and $\theta$, but not on $\phi$. For conciseness, the dependance on $r$ and $h$ is omited in the notation for $K$ and in the notations for other variables hereafter. Let us introduce the ratios:

$$
x=\frac{t}{2 r}, \quad y=\frac{h}{t} .
$$

The two variables $x$ and $y$, not to be confounded with Cartesian coordinates, will be used preferentialy to $t, h$ and $r$. Furthermore, the variable $t$ as argument of a function will be replaced indifferently by $x$ or $y$. For instance $K(\theta, t)$ is also denoted $K(\theta, x)$. 
The expression for the covariogram $K$ is derived from the formula of a covariogram of a disk in the plane and reads [9]:

$$
\begin{aligned}
K(\theta, t) & =\widetilde{K}(\theta, x) H(\theta, t), \\
\widetilde{K}(\theta, x) & =4 r^{3}\left(\frac{h}{2 r}-x \sin \theta\right)\left[\cos ^{-1}(x \cos \theta)-x \cos \theta \sqrt{1-x^{2} \cos ^{2} \theta}\right],
\end{aligned}
$$

where $H(\theta, t)=1$ when $\theta$ and $t$ are such that the two cylinders $\boldsymbol{C}$ and $\boldsymbol{C}^{\prime}$ intersect, and 0 otherwise. More precisely:

$$
H(\theta, t)=\left\{\begin{array}{l}
1 \text { if } t \leq t_{\max }, \\
0 \text { otherwise, }
\end{array} \quad t_{\max }=\left\{\begin{array}{l}
\frac{2 r}{\cos \theta} \text { if } \theta \in\left[0 ; \tan ^{-1} \frac{h}{2 r}\right], \\
\frac{h}{\sin \theta} \text { if } \theta \in\left[\tan ^{-1} \frac{h}{2 r} ; \frac{\pi}{2}\right] .
\end{array}\right.\right.
$$

The term $\cos ^{-1}$ in Eq. (4) refers to the inverse cosine function, also denoted arccos and $\tan ^{-1}$ in (5) denotes the inverse tangent function. Likewise, $\sin ^{-1}$ hereafter is used to denote the inverse sine function.

The following section is concerned by the normalized mean covariogram:

$$
k(t)=\frac{1}{4 \pi} \int_{0}^{2 \pi} \mathrm{d} \phi \int_{-\pi / 2}^{\pi / 2} \mathrm{~d} \theta \frac{K(\theta, t)}{h \pi r^{2}} \cos \theta=\int_{0}^{\pi / 2} \mathrm{~d} \theta \frac{K(\theta, t)}{h \pi r^{2}} \cos \theta,
$$

where the mean is taken over all directions on the sphere, assuming the distribution of orientations is uniform on the latter. Here, the covariogram $K$ is normalized by the cylinder volume and surface area of the unit sphere so that the function $k(t)=1$ when $t=0$ and $k(t)=0$ when $t=\infty$. Like $K$, the quantity $k$ depends on $r \geq 0$ and $h \geq 0$.

\section{MEAN COVARIOGRAM OF A CYLINDER}

In this section, the isotropized covariogram of a cylinder with circular cross-section is given. We refer to $[6-8,23]$ where this problem has been studied in details.

\section{A. Prolate cylinders}

In this section, we compute the limit $k_{\infty}(t)$ of $k(t)$ when $h \rightarrow \infty$ with $r$ and $t$ fixed. The condition $t \leq t_{\max }$ (Eq. 5) reduces to $x \cos \theta \leq 1$ which is satisfied for all $\theta$ when $x<1$ and for $\theta>\cos ^{-1}(1 / x)$ when $x>1$. Therefore the term $K$ can be replaced by $\widetilde{K}$ in (6) provided the integration is carried out in the intervals $[0 ; \pi / 2](x<1)$ and $\left[\cos ^{-1}(1 / x) ; \pi / 2\right](x>1)$ :

$$
k_{\infty}(x)= \begin{cases}\lim _{h \rightarrow \infty} \frac{1}{h \pi r^{2}} \int_{0}^{\pi / 2} \mathrm{~d} \theta \widetilde{K}(\theta, x) \cos \theta, & \text { if } x<1, \\ \lim _{h \rightarrow \infty} \frac{1}{h \pi r^{2}} \int_{\cos ^{-1}(1 / x)}^{\pi / 2} \mathrm{~d} \theta \widetilde{K}(\theta, x) \cos \theta, & \text { if } x>1\end{cases}
$$

Observe however that, when $x>1, \widetilde{K}(\theta, t)$ is purely imaginary in the domain $0<\theta<$ $\cos ^{-1}(1 / x)$. Accordingly, it is sufficient to integrate in the interval $[0 ; \pi / 2]$ instead of $\left[\cos ^{-1}(1 / x) ; \pi / 2\right]$, provided the imaginary part of the integral is discarded:

$$
k_{\infty}(x)=\lim _{h \rightarrow \infty} \frac{1}{h \pi r^{2}} \operatorname{Re}\left\{\int_{0}^{\pi / 2} \mathrm{~d} \theta \widetilde{K}(\theta, x) \cos \theta\right\},
$$


where Re stands for the real part of complex numbers. Replacing $K$ with its expression (4) and expanding the integrand at first order in $1 / h$ yields:

$$
k_{\infty}(x)=\frac{2}{\pi} \operatorname{Re}\left\{\int_{0}^{\pi / 2} \mathrm{~d} \theta \cos \theta\left[\cos ^{-1}(x \cos \theta)-x \cos \theta \sqrt{1-x^{2} \cos ^{2} \theta}\right]\right\} .
$$

As seen above, $k_{\infty}$ depends on $t$ and $r$ through $x=t /(2 r)$ only. The integral in (9) is readily computed in the complex domain using a symbolic calculator [27]. The expression depends on the complete elliptic integrals of the first and second kind, denoted $F$ and $E$ respectively and defined by:

$$
F(z)=\int_{0}^{\pi / 2} \frac{\mathrm{d} u}{\sqrt{1-z \sin ^{2} u}}, \quad E(z)=\int_{0}^{\pi / 2} \mathrm{~d} u \sqrt{1-z \sin ^{2} u} .
$$

The above functions $F$ and $E$ are real-valued when $z \leq 1$ and complex with non-zero real and imaginary parts when $z>1$. We refer to the online ressources [25] (and references therein) for an overview of their properties. Note that the elliptic functions are usually defined by $z \rightarrow F(\sqrt{z}), E(\sqrt{z})$. In this work we follow the notation used in [27].

The elliptic functions $F$ and $E$ appear in the integration of both the square root and inverse cosine terms in (9). For instance, when $x<1$, the $\cos ^{-1}$ term is integrated by parts as follows:

$$
\begin{aligned}
\int_{0}^{\pi / 2} \mathrm{~d} \theta \cos ^{-1}(x \cos \theta) \cos \theta & =\int_{0}^{\pi / 2} \frac{x \sin ^{2} \theta \mathrm{d} \theta}{\sqrt{1-x^{2} \cos ^{2} \theta}}=\left(x-\frac{1}{x}\right) F\left(x^{2}\right)+\frac{E\left(x^{2}\right)}{x} \\
& =\frac{\sqrt{1-x^{2}}}{x} \operatorname{Im}\left\{E\left(\frac{1}{1-x^{2}}\right)\right\},
\end{aligned}
$$

where Im denotes the imaginary part of complex numbers. The final formula for $k(x)$ reads, after simplification in the regions $x<1$ and $x>1$ :

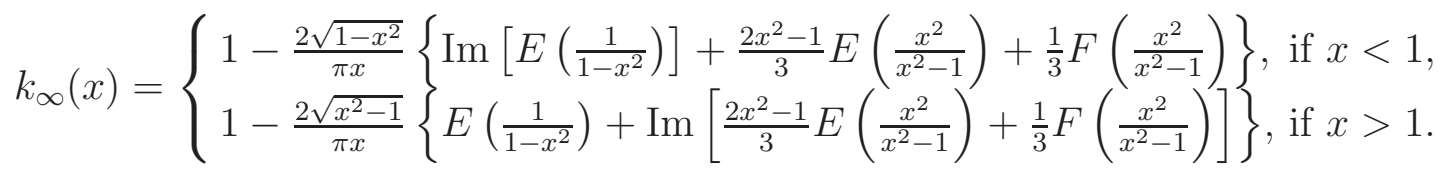

The function $k_{\infty}$ is represented in Fig. 1a (top curve).

\section{B. Cylinders of arbitrary aspect ratio}

This section is devoted to the general case, i.e. finite $h$ and $r$. The intervals of integration for $\theta$ are required for computing (6). Take first $h<2 r$ and examine the condition $t \leq t_{\max }$ in (5). The quantity $K$ in (4) is non-zero in the following intervals for $\theta$ (recall that $y=h / t$ ):

$$
\theta \in \begin{cases}{[0 ; \pi / 2]} & \text { if } t<h \\ {\left[0, \sin ^{-1}(y)\right]} & \text { if } h<t<2 r \\ {\left[\cos ^{-1}(1 / x), \sin ^{-1}(y)\right]} & \text { if } 2 r<t\end{cases}
$$

Whereas, when $h>2 r, K$ is non-zero if:

$$
\theta \in \begin{cases}{[0 ; \pi / 2]} & \text { if } t<2 r \\ {\left[\cos ^{-1}(1 / x), \pi / 2\right]} & \text { if } 2 r<t<h \\ {\left[\cos ^{-1}(1 / x), \sin ^{-1}(y)\right]} & \text { if } h<t\end{cases}
$$


As in Sec. (III A), $\widetilde{K}$ is purely-imaginary when $\theta \in\left[0 ; \cos ^{-1}(1 / x)\right]$, so one may carry out the integration in (6) along intervals $[0 ; a]$ with $a=\pi / 2$ or $a=\sin ^{-1}(y)$ depending on conditions precised in (13) and (14). We are left with the evaluation of the following integral:

$$
k(x, y)=\operatorname{Re}\left\{\int_{0}^{a} \mathrm{~d} \theta \frac{\widetilde{K}(\theta, x) \cos \theta}{h \pi r^{2}}\right\} .
$$

Now according to (13) and (14), $a=\pi / 2$ when $y>1(t<h)$ and $a=\sin ^{-1} y$ when $y<1$ $(t>h)$. We first examine the case $a=\pi / 2$, i.e. $y>1$. Equation (15) takes the form:

$$
\begin{aligned}
k(x, y)= & k_{y>1}(x, y)=k_{\infty}(x) \\
& -\frac{2}{\pi y} \operatorname{Re}\left\{\int_{0}^{\pi / 2} \mathrm{~d} \theta \cos (\theta) \sin (\theta)\left[\cos ^{-1}(x \cos \theta)-x \cos \theta \sqrt{1-x^{2} \cos ^{2} \theta}\right]\right\},
\end{aligned}
$$

where we have identified $k_{\infty}(x)$, the covariogram for $h=\infty$ given in (12). The integral in (16) is computed using a software for symbolic computations, and, after rearranging the terms:

$$
k_{y>1}(x, y)=k_{\infty}(x)-\frac{1}{8 x^{2} y}+\frac{H^{\prime}(x)}{2 y \pi}\left[\left(\frac{1}{2 x}+x\right) \sqrt{1-x^{2}}+\left(\frac{1}{2 x^{2}}-2\right) \cos ^{-1} x\right],
$$

where $H^{\prime}$ is the step function:

$$
H^{\prime}(x)=\mathbb{1}_{[1 ;+\infty]}= \begin{cases}1 & \text { if } x<1 \\ 0 & \text { if } x>1\end{cases}
$$

As expected, the quantity between square brackets in (17) is real when $x<1$ and so is $k(x, y)$ for all $x$.

We now consider the case $y<1(t>h)$ and the integral in (15) with $a=\sin ^{-1} y$. The symbolic calculator [27] provides a lengthy formula for the solution. After simplification, the expression takes different forms for $x<1$ and $x>1$. For $x>1$ and $y<1$, the covariogram $k$ reads:

$$
\begin{aligned}
k_{y>1}(x, y)= & \frac{4 \sqrt{x^{2}-1}}{3 \pi x} \operatorname{Im}\left[F\left(\sin ^{-1} y \mid \frac{x^{2}}{x^{2}-1}\right)-\left(1+x^{2}\right) E\left(\sin ^{-1} y \mid \frac{x^{2}}{x^{2}-1}\right)\right] \\
& +\left(\frac{y}{\pi}+\frac{4 x^{2}-1}{4 \pi y x^{2}}\right) \cos ^{-1}\left(x \sqrt{1-y^{2}}\right) \\
& -\frac{1}{2 \pi}\left(\frac{x y}{3}+\frac{1}{2 x y}+\frac{x}{y}\right) \sqrt{1-y^{2}} \sqrt{1-x^{2}\left(1-y^{2}\right)} .
\end{aligned}
$$

The functions of two variables $F(\phi \mid z)$ and $E(\phi \mid z)$ are the incomplete elliptic integrals of the first and second kind, respectively, defined by [26]:

$$
E(\phi \mid z)=\int_{0}^{\phi} \mathrm{d} u \sqrt{1-z \sin ^{2} u}, \quad F(\phi \mid z)=\int_{0}^{\phi} \frac{\mathrm{d} u}{\sqrt{1-z \sin ^{2} u}} .
$$

The complete elliptic integrals in (10) are special cases:

$$
E(z)=E\left(\frac{\pi}{2} \mid z\right), \quad F(z)=F\left(\frac{\pi}{2} \mid z\right) .
$$




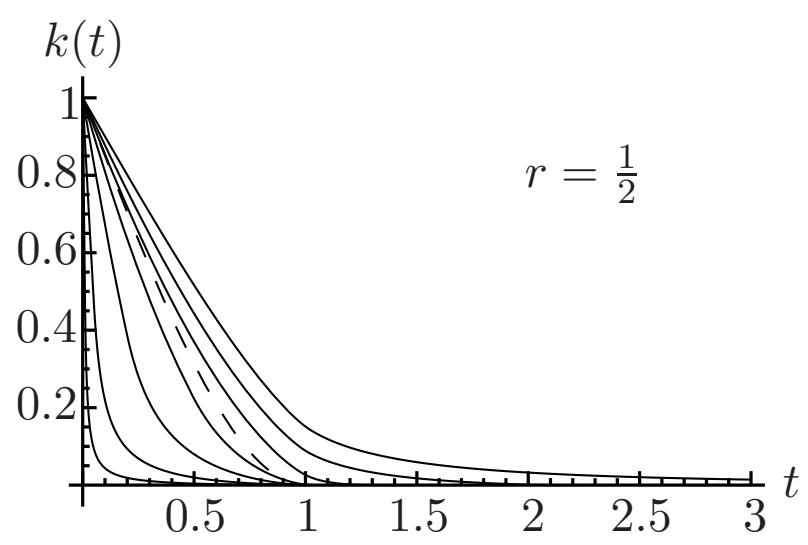

(a)

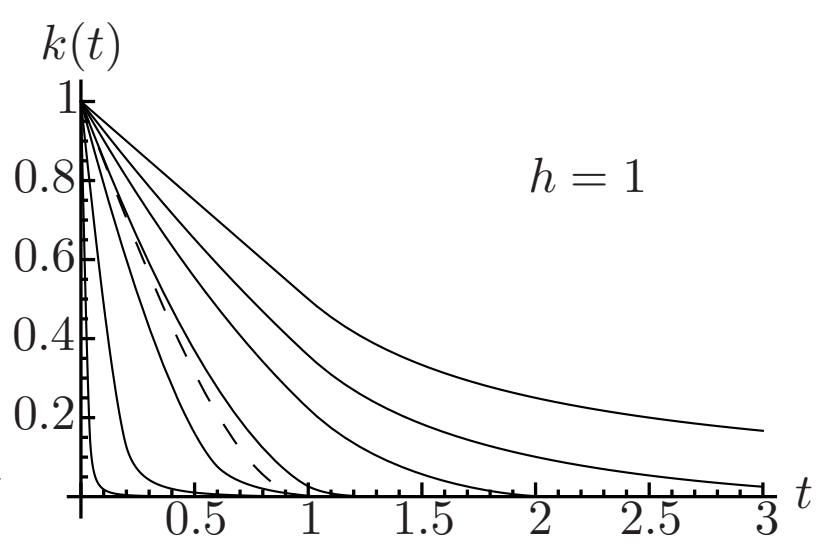

(b)

Figure 1: Normalized mean covariogram $k(t)$ as a function of $t$ : (a) with $r=1 / 2$ fixed and increasing height $h=0.01,0.05,0.2,0.5,1,2,+\infty$ (bottom to top, solide lines); (b) with $h=1$ fixed and increasing radius $r=0.02,0.1,0.3,0.5,1,2,+\infty$ (bottom to top, solide lines). Dashed lines: covariogram of a sphere of diameter 1.

The incomplete elliptic integrals are real-valued if $z \sin ^{2} \phi<1$. Note that this is never the case for the arguments of $E$ and $F$ in (19), so that the two functions have complex values. Note also that, owing to $t \leq \sqrt{h^{2}+4 r^{2}}$ :

$$
x \sqrt{1-y^{2}}<1, \quad 1-x^{2}\left(1-y^{2}\right)<1,
$$

and so $k_{y>1}(x, y)$ in $(19)$ is real. Now, when $x, y<1$, one finds for $k(x, y)$ :

$$
\begin{aligned}
k_{y<1}(x, y)= & \frac{4 \sqrt{1-x^{2}}}{3 \pi x}\left[F\left(\sin ^{-1} y \mid \frac{x^{2}}{x^{2}-1}\right)-\left(1+x^{2}\right) E\left(\sin ^{-1} y \mid \frac{x^{2}}{x^{2}-1}\right)\right] \\
& +\left(\frac{y}{\pi}+\frac{5 x^{2}-2}{4 \pi y x^{2}}\right) \cos ^{-1}\left(x \sqrt{1-y^{2}}\right)+\frac{2-5 x^{2}}{4 \pi y x^{2}} \cos ^{-1} x \\
& -\frac{1}{2 \pi}\left(\frac{x y}{3}+\frac{1}{2 x y}+\frac{x}{y}\right) \sqrt{1-y^{2}} \sqrt{1-x^{2}\left(1-y^{2}\right)}+\frac{1+2 x^{2}}{4 \pi y x} \sqrt{1-x^{2}} \\
& +\frac{1-x^{2}}{4 \pi y x^{2}} \cos ^{-1}\left(x^{2} \sqrt{1-y^{2}}+\sqrt{1-x^{2}} \sqrt{1-x^{2}\left(1-y^{2}\right)}\right) .
\end{aligned}
$$

Note the similarities with the formula for $x>1$ in (19). Also, all three expressions inside $\cos ^{-1}$ in the above are comprised between 0 and 1 and so $k(x, y)$ is real.

To summarize, the exact expression for $k(x, y)$ is given by (17) and (12) when $y>1$, by (19) when $y<1<x$, and by (23) when $x<1$ and $y<1$. The covariogram is plotted as a function of $t$ in Fig. (1a) and (1b) for various values of $r$ and $h$ (solid lines), and compared with that of a sphere (dotted lines).

An asymptotic expansion of $k(t)$ when $t \rightarrow 0$ is now carried out. Use the expansion $(m \rightarrow 0):$

$$
F(\phi \mid m)=\phi+\frac{\sin (2 \psi)-2 \psi}{8} m+O\left(m^{2}\right), \quad E(\phi \mid m)=\phi+\frac{\sin (2 \psi)-2 \psi}{8} m+O\left(m^{2}\right),
$$


to find:

$$
k_{\infty}(t)=1-\frac{t}{2 r}+O\left(t^{3}\right), \quad k(t)=1-\frac{1}{2}\left(\frac{1}{r}+\frac{1}{h}\right) t+\frac{2}{3 \pi h r} t^{2}+O\left(t^{3}\right), \quad t \rightarrow 0 .
$$

As expected the derivative of $k(t)$ at $t=0$ is equal (up to the sign) to the surface/volume ratio of the cylinder.

The second-order derivative of $k(t)$ becomes infinite at the point $t=h[6]$. Connections between the second-order derivative of the covariogram of a compact set and and its singular points have been previously noticed in the plane [5].

\section{Oblate cylinders}

Consider now the limit $r \rightarrow \infty$ with $h$ fixed so that $x \rightarrow 0$ and $y$ remains finite. We take the limit in the formula (23) when $y<1$ and (17) when $y>1$. The covariogram $k$ depends of $y$ only and reads:

$$
k_{0}(y)= \begin{cases}1-\frac{1}{2 y}, & \text { if } y>1 \\ \frac{y}{2}, & \text { if } y<1\end{cases}
$$

Note that, in the limit $r \rightarrow \infty$ the cylinders amount to infinite layers enclosed between two planes. The same asymptotic limit would accordingly be recovered when considering cylinders with other non-circular cross-section. This is in contrast to the prolate case (Sec. III A), where the covariogram depends on the shape of the cylinders cross-section.

\section{Approximate formulas}

We now give approximate expressions of the exact solutions which do not involve Elliptic functions. Such simpler expressions are useful for material applications, in particular for identifying random models, when a high accuracy is not required.

We first consider the domain $r \gg h$. The exact solution is given by (26) when $r=\infty$ and by (17) and (23) when $r<\infty$. We first let $r \rightarrow \infty$ and $t \rightarrow \infty$ with $h$ and the ratio $t / r$ fixed, and expand (23) to order $O\left(r^{-3}\right)$. In the region $t<h$, i.e. $y>1$, we let $t \rightarrow 0$ and compute an expansion to $O\left(t^{3}\right)$ with $r, h$ fixed. In the region $t>2 r$, we set $k \approx 0$. One obtains:

$$
k(t) \approx k_{r \gg h}(t)=\left\{\begin{array}{cc}
1-\frac{1}{2}\left(\frac{1}{h}+\frac{1}{r}\right) t+\frac{2 t^{2}}{3 \pi r h}+\frac{t^{3}}{64 r^{3}}, & \text { if } t<h, \\
\frac{h}{\pi t} \cos ^{-1}\left(\frac{t}{2 r}\right)+\left(\frac{h^{2}}{6 t^{2}}-1\right) \frac{h}{2 \pi r} \sqrt{1-\frac{t^{2}}{4 r^{2}}}, & \text { if } h<t<2 r, \\
0 & \text { if } t>2 r .
\end{array}\right.
$$

The expansion above is continuous except at points $t=h$, and is exact in the limit $r=\infty$ only. It turns out, however, that formula (27) is a very good approximation of the exact covariogram for $r>h$. The maximal error $\sup _{t}\left|k(t)-k_{r \gg h}(t)\right|$ between $(27)$ and the exact result is attained when $t \lesssim h$ whenever $r>h$. As expected, this error decreases and tends to 0 when $r / h \rightarrow \infty$. It is about $0.5 \%$ for $r=h$ and $0.1 \%$ for $r=4 h$ (Fig. 2). The error is much smaller at points $t \not z h$ : for instance, the mean error $\left\langle\left|k(t)-k_{r \gg h}(t)\right|\right\rangle_{t}$ is $0.1 \%$ and $0.004 \%$ when $r=h$ and $r=4 h$ respectively. Approximation (27) is good in the region 


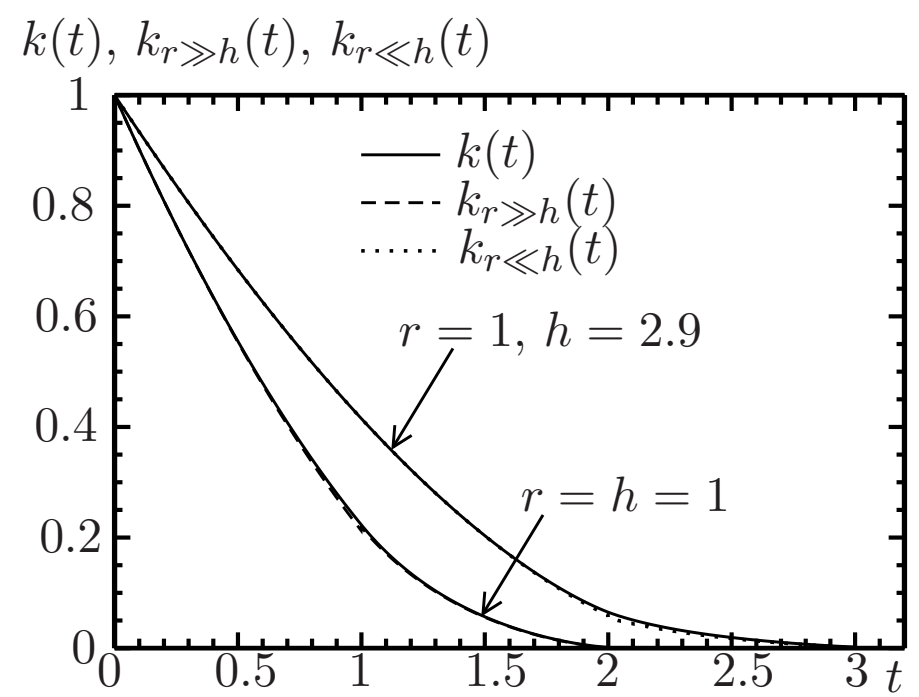

Figure 2: Mean normalized covariogram $k$ of a cylinder: comparison between the exact result (solid line) and approximations (27) and (28) (dotted lines) when $r=h=1$ and $r=1, h=2.9$, respectively.

$h / 2<r<h$. The errors are about $\sup _{t}\left|k-k_{r \gg h}\right|=2.6 \%$ and $\left\langle\left|k-k_{r \gg h}\right|\right\rangle_{t}=0.6 \%$ when $r=h / 2$.

We now consider the domain $h \gg r$ and compute an approximation of $k(t)$ valid in the region $h \gg r$. This task is more difficult than in the oblate case $h \ll r$ because the exact result for $h=\infty$ involves elliptic functions. In the domain $2 r<t<h$, we let $h$ and $t \rightarrow \infty$ with $r$ and the ratio $h / t$ fixed and expand (17) to $O\left(h^{-4}\right)$. We use the same expression in the domain $t>h$, as long as the latter is positive and 0 otherwise. In the domain $t<2 r$, we expand (19) when $h, t \rightarrow \infty$ with $r$ and the ratio $h / t$ fixed, to $O\left(h^{-4}\right)$. One finds:

$k(t) \approx k_{r \ll h}(t)=\left\{\begin{array}{cr}1-\frac{t}{2 r}\left[1-\frac{1}{2}\left(\frac{t}{4 r}\right)^{2}-\frac{1}{4}\left(\frac{t}{4 r}\right)^{4}\right]-\frac{t}{2 h}+\frac{2 t^{2}}{3 \pi h r}\left(1-\frac{t^{2}}{40 r^{2}}\right), & t<2 r, \\ \max \left\{0 ; \frac{r^{2}}{2 t^{2}}\left(1-\frac{t}{h}+\frac{r^{2}}{2 t^{2}}\right)\right\}, & t>2 r .\end{array}\right.$

We emphasize that, contrary to (27), the expressions above are not asymptotically correct in the limit $h \rightarrow \infty$. Also, approximation $k_{r \ll h}(t)$ is discontinuous at $t=2 r$. The maximal error $\sup _{t}\left|k(t)-k_{r \ll h}(t)\right|$ is attained at $t \gtrsim 2 r$, is constant and equal to about $1.1 \%$ for $h>2 r$. In the domain $1.6 r<h<2 r$, the maximal error is less than $1.4 \%$ (Fig. 2).

To summarize, a good approximation of the covariogram is given by (27) when $h<1.6 r$ and by (28) when $h>1.6 r$ with a maximal absolute error of $1.8 \%$.

\section{BOOLEAN MODEL OF CYLINDERS}

In this section, we consider a Boolean model [17] of cylinders with radius $r$ and height $h$. The Boolean model is defined by an homogeneous Poisson point process of intensity $\psi$ (average number of points per unit area). A cylinder $\boldsymbol{C}$ oriented in a random direction, uniformly distributed on the sphere, is implanted on each Poisson point. The cylinder 
volume fraction, denoted $p$, is linked to the Poisson intensity through [16]:

$$
\log (p)=-\psi \pi h r^{2} .
$$

Hereafter, we examine the integral range of Boolean random sets made of cylinders. The Boolean model, denoted $\mathbf{B}$ is the union of all cylinders $\boldsymbol{C}$, implanted at Poisson points so that cylinders may interpenetrate. Its characteristic function is denoted $\chi_{\mathbf{B}}$.

\section{A. Integral range}

The covariance $C(t)$ of the Boolean model of cylinders $\mathbf{B}$ reads:

$$
C(t)=\{z \in \mathbf{B}, z+\mathbf{t} \in \mathbf{B},|\mathbf{t}|=t\}=2 p-1+(1-p)^{2-k(t)} .
$$

Its integral range is defined by [17]:

$$
A_{3}=\frac{1}{p(1-p)} \int_{0}^{\infty} \mathrm{d} t 4 \pi t^{2}\left[C(t)-p^{2}\right]=\frac{4 \pi(1-p)}{p} \int_{0}^{\infty} \mathrm{d} t t^{2}\left[\mathbf{e}^{-k(t) \log (1-p)}-1\right],
$$

where the integrand $C(t)-p^{2}$ is the centered covariance. For cylinders the centered covriance is identically zero for $t \geq t_{c}$ where $t_{c}=\sqrt{4 r^{2}+h^{2}}$ is the maximum length of a chord in the cylinder. The integral range is useful to quantify the representative volume element for the set B. Using the exact expression derived in Sec. (IIIB), we compute the normalized covariogram:

$$
\widetilde{A}_{3}=\frac{A_{3}}{\pi h r^{2}},
$$

for $r=1 / 2$ (Fig. 3). The latter is equal to 1 when $p=0$. Remark that the integral range is very close, but not equal, to that of a Boolean model of sphere of diameter 1:

$$
\widetilde{A}_{3}^{\text {sphere }}=\frac{6}{\pi p} \int_{0}^{1} \mathrm{~d} t\left[(1-p)^{3 t / 2-t^{3} / 2}-1+p\right] .
$$

When $p=1 / 2$, the maximum value of $A_{3}$ is found to occur for $h$ very slightly smaller than 1 (Fig. 4). This value is still smaller than $\widetilde{A}_{3}^{\text {sphere }}$. Furthermore, numerical computations show that the normalized integral range $A_{3}$ is almost unchanged when $h$ is changed to $1 / h$ (Fig. 3).

\section{B. Variances and representative volume element: prolate and oblate cylinders} $V$ :

Assume $p_{W}$ is the measure of the volume fraction of cylinders over a domain $W$ of volume

$$
p_{W}=\frac{L_{3}(\mathbf{B} \cap V)}{V}=\int_{W} \mathrm{~d} u \chi_{\mathbf{B}}(u) .
$$

Denote $\bar{p}_{W}$ the mean of the estimates $p_{W_{i}}$ taken over $N$ independant realizations $W=W_{1}$, $\ldots, W_{N}$ of volume $V$. The variance $D_{\mathbf{B}}^{2}(V)$ of the estimates $p_{W_{i}}$ is given by:

$$
D_{\mathbf{B}}^{2}(V)=\frac{1}{N} \sum_{i=1}^{N}\left[\frac{1}{V} \int_{W_{i}} \mathrm{~d} u \chi_{\mathbf{B}}(u)-\bar{p}_{W}\right]^{2}=\frac{1}{N} \sum_{i=1}^{N}\left[\left(\frac{1}{V} \int_{W_{i}} \mathrm{~d} u \chi_{\mathbf{B}}(u)\right)^{2}-\bar{p}_{W}^{2}\right] .
$$




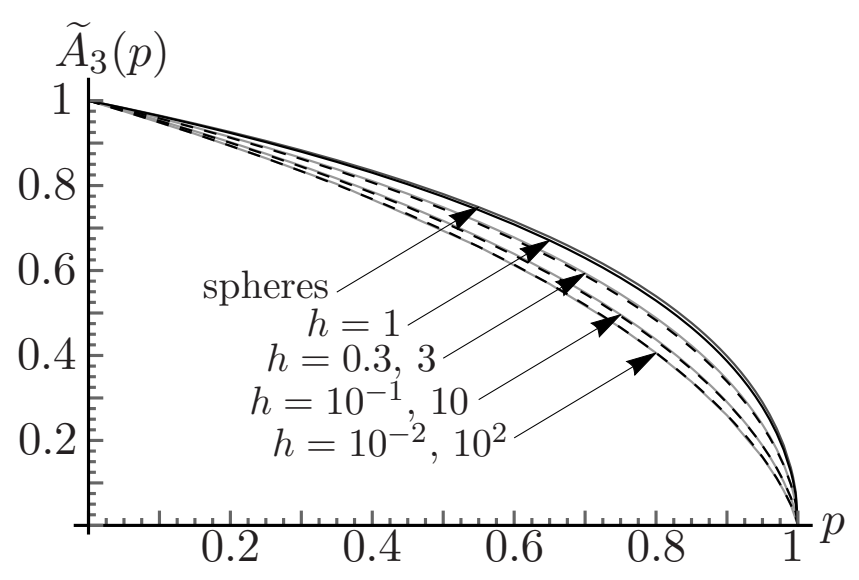

Figure 3: Normalized integral range $\widetilde{A}_{3}(p)$ in a Boolean model of cylinders with radius $r=1 / 2$ as a function of the volume fraction $p$ of cylinders, with varying cylinder heights $h=3,10,10^{2}$ (solid grey lines, top to bottom), $1 / 3,10^{-1}, 10^{-2}$, (dotted black lines, top to bottom) and $h=1$ (solid black line). Dark-grey solid line on top: normalized integral range for a Boolean model of spheres of diameter 1.

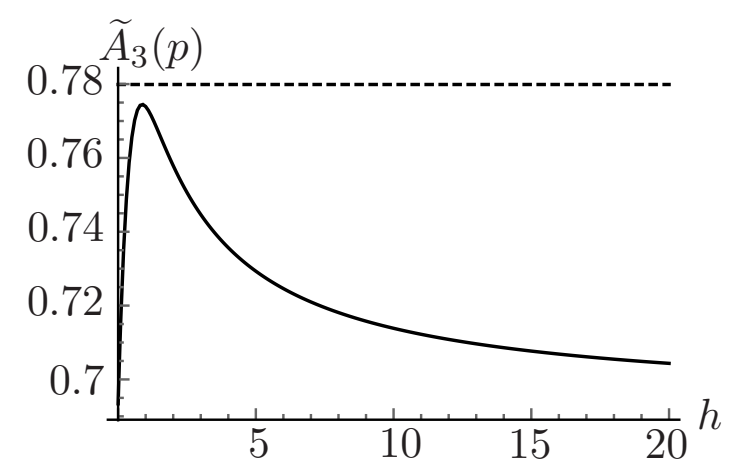

Figure 4: Normalized integral range $\widetilde{A}_{3}(p)$ in a Boolean model of cylinders with radius $r=1 / 2$ and volume fraction $p=1 / 2$ as a function of the cylinders height $h$ (solid line). Dashed line: normalized integral range for a Boolean model of spheres of diameter 1.

Taking $N$ large and $\bar{p}_{W} \approx p$ in the above:

$$
D_{\mathbf{B}}^{2}(V)=\frac{1}{N V^{2}} \sum_{i=1}^{N} \iint_{u, v \in W_{i}} \mathrm{~d} u \mathrm{~d} v\left[\chi_{\mathbf{B}}(u) \chi_{\mathbf{B}}(v)-p^{2}\right] .
$$

Take $W_{i} \rightarrow \mathbb{R}^{3}$ and make use of the variable change $t=v-u$. The double integral amounts to an integral of the centered covariance function and so:

$$
D_{\mathbf{B}}^{2}(V)=\frac{1}{V} \int_{\mathbf{R}^{3}} \mathrm{~d} t\left[C(t)-p^{2}\right]=p(1-p) \frac{A_{3}}{V},
$$

a result first derived by Matheron [17]. The asymptotic expansion (37) is actually valid for volumes $V$ much larger than $A_{3}$ and so requires that $A_{3}$ (and $t_{c}$ ) is finite. The latter shows that, when $V \gg A_{3}$, the volume $W$ acts as $n$ independant domains of volume $A_{3}$ with $n=W / A_{3}$. Thus the volume $A_{3}$ is said to be "representative" of the Boolean model B. Note however that the variance $D_{\mathbf{B}}^{2}(V)$ also depends on the point variance $p(1-p)$. 
When $A_{3}$ is infinite, a scaling law different from (37) is expected [12]. For prolate and oblate cylinders, the theory respectively predicts [10]:

$$
D_{\mathbf{B}}^{2}(W) \sim \frac{\alpha}{V^{2 / 3}}, \quad D_{\mathbf{B}}^{2}(W) \sim \frac{\alpha^{\prime}}{V^{1 / 3}}, \quad V \gg A_{3},
$$

where $\alpha, \alpha^{\prime}$ are prefactors. The scaling laws above indicate a slower decrease of the variance with respect to the volume than in (37), due to infinite correlation lengths.

For prolate and oblate cylinders, the behavior of $D_{\mathbf{B}}^{2}(W)$ should be linked to that of the integral of the covariance $C(t)$ when $t \rightarrow \infty$. Consider first the asymptotic behavior of $k(t)$ when $t \rightarrow \infty$ in the cases $h=\infty$ and $r=\infty$. Making use of the expansions for $\phi \rightarrow 0$ :

$$
\begin{aligned}
& F(\phi \mid m)=\phi+\frac{m \phi^{3}}{6}+\frac{m(9 m-4) \phi^{5}}{120}+O\left(\phi^{7}\right), \\
& E(\phi \mid m)=\phi-\frac{m \phi^{3}}{6}+\frac{m(4-3 m) \phi^{5}}{120}+O\left(\phi^{7}\right),
\end{aligned}
$$

one finds, when $t \rightarrow \infty$ :

$$
k_{\infty}(t)=\frac{r^{2}}{2 t^{2}}+O\left(\frac{1}{t^{4}}\right), \quad(\text { if } h=+\infty), \quad k_{0}(t)=\frac{h}{2 t}+O\left(\frac{1}{t^{2}}\right), \quad(\text { if } r=+\infty) .
$$

For prolate and oblate cylinders, the integral of the centered covariance on a spherical domain of radius $\ell$ diverges as:

$$
A_{3}(\ell)=\int_{t \leq \ell}\left(4 \pi t^{2}\right) \mathrm{d} t\left[C(t)-p^{2}\right] \sim \begin{cases}-\frac{2 \pi(1-p)}{p} r^{2} \log (1-p) \ell, & \text { if } h \rightarrow \infty, \\ -\frac{\pi(1-p)}{p} h \log (1-p) \ell^{2}, & \text { if } r \rightarrow \infty .\end{cases}
$$

We remark that $A_{3}(\ell) / \ell^{3}$ behave as $\sim V^{-2 / 3}$ and $\sim V^{-1 / 3}$ respectively for prolate and oblate cylinders, where $V$ is the sphere of radius $\ell$. This qualitatively explains the expansions (38).

However, the variable change $t=v-u$ leading to (37) can not be directly carried out for finite domains $W_{i}$ and infinite integral range. We derive it here for spherical domains $W_{i}$ of radius $\ell$. One needs to compute the probability $\mathrm{d} P(t, \ell)$ that two points $A$ and $B$ in a sphere are separated from a distance in the interval $[t ; t+\mathrm{d} t]$. We first fix $A$, a point at a distance $0<a<\ell$ of the sphere center. The volume of points at a distance in the interval $[t ; t+\mathrm{d} t]$ from $A$ is given by:

$$
\mathrm{d} V_{t}= \begin{cases}4 \pi t^{2} \mathrm{~d} t, & \text { if } t<\ell-a, \\ 2 \pi t \mathrm{~d} t\left(t-\frac{a}{2}+\frac{\ell^{2}-t^{2}}{2 a}\right), & \text { if } \ell-a<t<\ell+a .\end{cases}
$$

Integrating over $a$, one finds the required probability:

$$
\mathrm{d} P(t, \ell)=\frac{3 \mathrm{~d} t}{\ell} \frac{t^{2}}{\ell^{2}}\left(1-\frac{t}{2 \ell}\right)^{2}\left(1+\frac{t}{4 \ell}\right), \quad 0 \leq t \leq 2 \ell .
$$

Replace now the term $\chi_{\mathbf{B}}(u) \chi_{\mathbf{B}}(v)$ in $(36)$ by $C(t)$ and integrate over $t$ :

$$
D_{\mathbf{B}}^{2}(V)=\frac{1}{N} \sum_{i=1}^{N} \frac{1}{V^{2}} \iint_{u, v \in W_{i}} \mathrm{~d} u \mathrm{~d} v\left[\chi_{\mathbf{B}}(u) \chi_{\mathbf{B}}(v)-p^{2}\right] \approx \int_{t \leq 2 \ell} \mathrm{d} P(t, \ell)\left[C(t)-p^{2}\right] .
$$




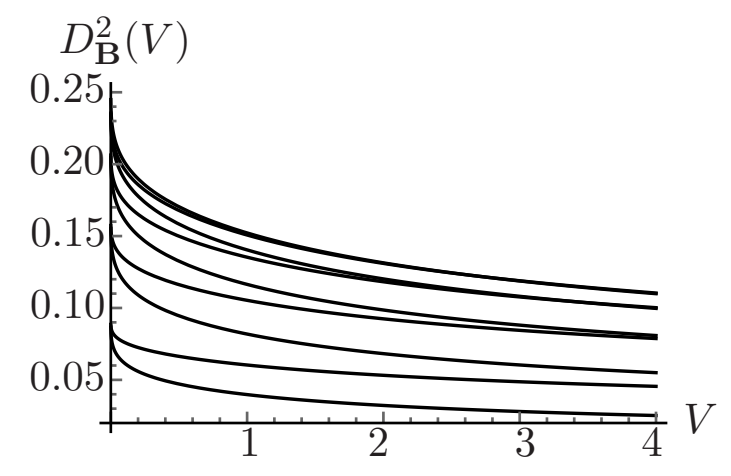

Figure 5: Variance $D_{\mathbf{B}}^{2}(V)$ of the estimate of the volume fraction measured in a spherical domain $W$ vs. volume size $V$ of the domain for oblate cylinders $(r=+\infty)$. The cylinders height is fixed to $h=1$. Top to bottom: volume fraction of cylinders $p=0.5,0.4,0.6,0.3,0.7,0.2,0.8,0.1,0.9$.

The integral above is analytically solvable as a closed-form expression, for oblate cylinders $(r=\infty)$. When $2 \ell>h$, the solution involves the exponential integral function $E_{i}(z)=$ $-\int_{-z}^{\infty} \mathbf{e}^{-s} / s \mathrm{~d} s$. The exact solution is a lengthy expression, which, for conciseness, is not given here. It is provided by the software Mathematica [27]. The variance $D_{\mathbf{B}}^{2}(V)$ is plotted as a function of $V$ for various volume fraction of cylinders in Fig. (5).

Taking the limit $\ell \rightarrow \infty$ and using the expansion (40) yields:

$$
\begin{aligned}
D_{\mathbf{B}}^{2}(V) \sim & \frac{-9(1-p)^{2} r^{2} \log (1-p)}{8 \ell^{2}}+O(1 / \ell)^{3}, \quad(h=+\infty), \\
D_{\mathbf{B}}^{2}(V) \sim & \frac{-3 h(1-p)^{2} \log (1-p)}{5 \ell} \\
& +\frac{9 h^{2}(1-p)^{2}[\log (1-p)]^{2}}{32 \ell^{2}}+O(1 / \ell)^{3}, \quad(r=+\infty) .
\end{aligned}
$$

when $\ell \rightarrow \infty$, with $V=(4 / 3) \pi \ell^{3}$. Note that the results above are identical up to a constant factor to those obtained by replacing $A_{3}$ in $(37)$ by $A_{3}(\ell)$ from (41).

\section{Variances and representative volume element: cylinders with finite height and radius}

This section is concerned with the behavior of $D_{\mathbf{B}}^{2}(V)$ for $r$ or $h$ large but not infinite. We first examine $h$ large. The integral in Eq. (44) is computed numerically for increasing values of $h=10,10^{2}, \ldots, 10^{4}$ with $r=1 / 2$ fixed (Fig. 6a). The data is compared to $h=\infty$ (solid line, top) and to the expansion (45) (dashed line, top). The variance $D_{\mathbf{B}}^{2}(V)$ asymptotically scales as $\sim 1 / V$ for very large $V$, as long as $h$ is finite. However, for $h \geq 10$, an intermediate regime appears where $D_{\mathbf{B}}^{2}(V) \sim 1 / V^{2 / 3}$. This scaling law occurs for $V \ll h^{3}$, i.e. $\ell \ll h$. It is very close to the asymptotic limit (45). The change between the two regimes takes place, as expected, when $\ell$ is of the same order as $h$. Numerical data indicates $\ell \approx 3 h$. A similar behavior happens for $r \gg h$ with $h=1$ fixed (Fig. 6b): when $\ell \gg r$, the scaling law $D_{\mathbf{B}}^{2}(V) \sim 1 / V$ holds, whereas $D_{\mathbf{B}}^{2}(V) \sim 1 / V^{1 / 3}$ when $\ell \ll r$. The change between the two 


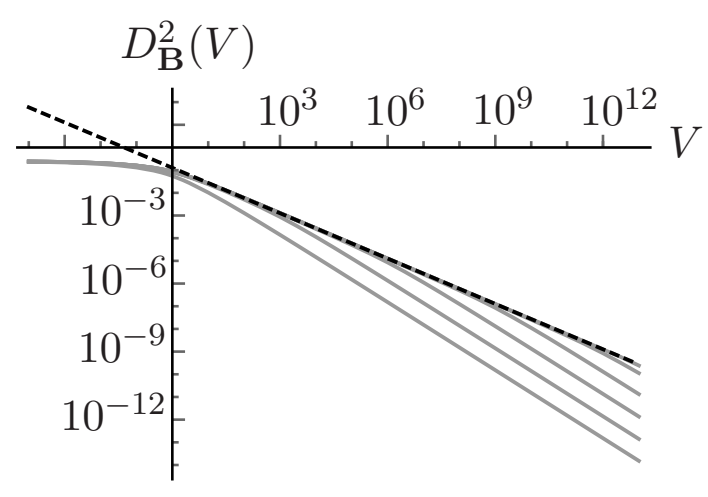

(a)

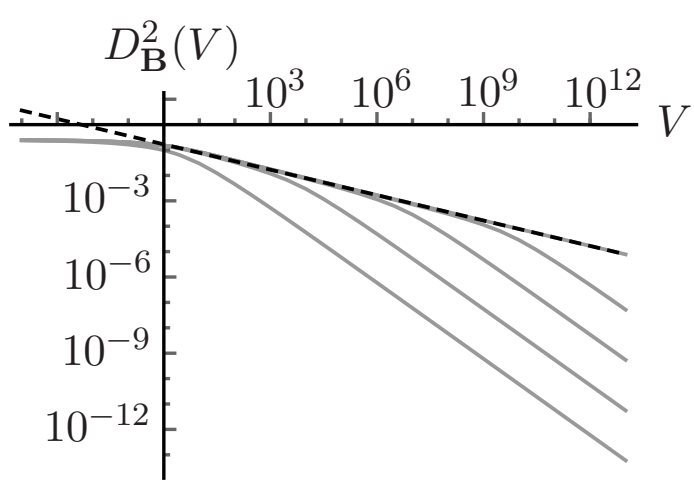

(b)

Figure 6: Variance $D_{\mathbf{B}}^{2}(V)$ of the estimate of the volume fraction measured in a spherical domain $W$ vs. volume size $V$ of the domain, in $\log -\log$ plot. The cylinders volume fration is fixed to $p=1 / 2$. Grey solid lines, bottom to top : (a) cylinders height $h=1,10,100,10^{3}, 10^{4}$, $\infty$ with radius fixed to $r=1 / 2$; (b) cylinders radius $r=1,10,100,10^{3}, \infty$ with height fixed to $h=1$. Dotted lines in black: expansions (45).

regimes occurs when $r \approx \ell$. Pluging $\ell=3 h$ and $\ell=r$ into (45) yields:

$$
\begin{array}{ll}
D_{\mathbf{B}}^{2}(V) \sim \frac{-27(1-p)^{2} \log (1-p) h r^{2}}{8 \ell^{3}}, & (\ell \gg h \gg r), \\
D_{\mathbf{B}}^{2}(V) \sim \frac{-3(1-p)^{2} \log (1-p) h r^{2}}{5 \ell^{3}}, & (\ell \gg r \gg h) .
\end{array}
$$

The two expansions above are in good agreement with the numerical data in Fig. (6).

\section{CONCLUSION}

In this work, the covariance and integral range of the Boolean model of cylinders have been computed using the geometrical covariogram of cylinders. As expected, the integral range takes the form of a divergent integral for flat (oblate) or highly-elongated (prolate) cylinders. This results in peculiar scaling laws of the variance of the measurement of the volume fraction of cylinders over subdomains of volume $V$. The latter variance scales as $\sim V^{-1 / 3}$ for the oblate and $\sim V^{-2 / 3}$ for the prolate type when $V \rightarrow \infty$. The lowest-order correction in the asymptotic expansion for the variance has been derived for domains of volume $V \rightarrow \infty$ with a spherical shape. For cylinders with finite (but large) aspect ratio, the scaling law $\sim V^{-1 / 3}$ or $\sim V^{-2 / 3}$ occurs in an intermediate region where $L=V^{1 / 3}$ is comprised between the lowest and highest dimensions of the cylinders. The classical scaling law $\sim 1 / V$ is recovered when $L$ is much larger than The classical scaling law $\sim 1 / V$ is recovered when $L$ is much larger than both cylinders dimensions. These results have implications regarding the effective thermal and elastic properties of cylinder models where similar powerlaws have been observed $[1,3]$. 


\section{Acknowledgments}

The author thanks C. Lantuéjoul for useful discussions and references.

[1] H. Altendorf, D. Jeulin, and F. Willot. Influence of the fiber geometry on the macroscopic elastic and thermal properties, Int. J. Sol. Struct. 51, 3807-3822 (2014).

[2] S. N. Chiu, D. Stoyan, W. S. Kendall, and J. Mecke. Stochastic Geometry and its applications, Third edition, Wiley, Chichester (2013).

[3] J. Dirrenberger, S. Forest, D. Jeulin. Towards gigantic RVE sizes for 3D stochastic fibrous networks, Int. J. Sol. Struct. 51, 359-376 (2014).

[4] E. Couka, F. Willot, and D. Jeulin. A mixed Boolean and deposit model for the modeling of metal pigments in paint layers, Im. An. Ster. 34, 125-134 (2015).

[5] X. Emery, and C. Lantuéjoul. Geometric covariograms, indicator variograms and boundaries of planar closed sets, Math. Geosc. 43, 905-927 (2011).

[6] W. Gille. The intercept length distribution density of a cylinder of revolution, Experimentelle Technik der Physik 35, 93-98 (1987).

[7] W. Gille. The chord length distributions of selected infinitely long geometric figures - connections to the field of small-angle scattering, Comp. Mat. Sci. 22, 318-332 (2001).

[8] W. Gille. Particle and Particle Systems Characterization, CRC Press, Boca Raton (2014).

[9] H. Harutyunyan, and V. Ohanyan. Covariogram of a cylinder, J. Contemp. Math. An. 49, 366-375 (2014).

[10] D. Jeulin. Power laws variance scaling of Boolean random varieties, Method. Comput. Appl. Prob. 18, 1065-1079 (2016).

[11] D. Jeulin, P. Monnaie, and F. Péronnet. Gypsum morphological analysis and modeling, Cem. Conc. Comp. 23, 299-311 (2001).

[12] C. Lantuéjoul. Ergodicity and integral range, J. Microscopy 161, 387-403 (1991).

[13] G. Matheron. Les variables régionalisées et leur estimation: une application de la théorie des fonctions aléatoires aux sciences de la nature, Masson, Paris (1965).

[14] G. Matheron. Éléments pour une théorie des milieux poreux, Masson, Paris (1967).

[15] G. Matheron. Ensembles fermés aléatoires, ensembles semi-markoviens et polyèdres poissoniens, Adv. Appl. Prob. 4, 508-541 (1972).

[16] G. Matheron. Random sets theory and its applications to stereology, J. Microscopy 95, 15-23 (1972).

[17] G. Matheron. Random sets and integral geometry, Wiley, New-York (1975).

[18] C. Peyrega, D. Jeulin, C. Delisée, and J. Malvestio. 3D morphological modelling of a random fibrous network, Im. Anal. Ster. 28, 129-141 (2009).

[19] C. Redenbach, and I. Vecchio. Statistical analysis and stochastic modelling of fibre composites, Composites Sci. Tech. 71, 107-112 (2011).

[20] K. Robb, O. Wirjadi, and K. Schladitz. Fiber orientation estimation from 3D image data: Practical algorithms, visualization, and interpretation. In $7^{\text {th }}$ International Conference on Hybrid Intelligent Systems (HIS), IEEE, 320-325 (2007).

[21] R. Schneider, and W. Weil. Stochastic and integral geometry, Springer-Verlag, Berlin, Heidelberg (2008).

[22] J. Serra. The Boolean model and random sets, Comp. Graph. Im. Proc. 12, 99-126 (1980). 
[23] H. S. Sukiasian, and W.Gille. "Relation between the chord length distribution of an infinitely long cylinder and that of its base", J. Math. Phys. 48, 53305-53312 (2007).

[24] H. Wang, A. Pietrasanta, D. Jeulin, F. Willot, M. Faessel, L. Sorbier, and M. Moreaud. Modelling mesoporous alumina microstructure with 3D random models of platelets, J. Microscopy 260, 287-301 (2015).

[25] E. Weisstein. MathWorld, http://mathworld.wolfram.com, accessed August 4, 2015.

[26] E. Weisstein. MathWorld, http://mathworld.wolfram.com/EllipticIntegralof theSecon dKind.html and http://mathworld.wolfram.com/EllipticIntegralof theSecondKind.ht $\mathrm{ml}$, accessed August 4, 2015.

[27] Wolfram Research, Inc. Mathematica software version 10.2 2015. Wolfram Research, Inc., Champaign, Illinois. 